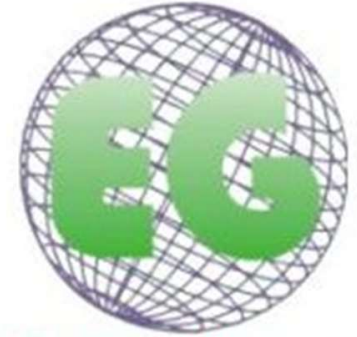

ISSN 1695-6141 $N^{\circ} 60$

\title{
Efecto del ejercicio cardiovascular sobre el autoconcepto en adultos mayores: ensayo clínico
}

Cardiovascular exercise effect in self-concept in seniors: clinical trial

\author{
Erick Landeros-Olvera ${ }^{1}$ \\ Joaquín Gil-Benitez ${ }^{2}$ \\ Claudia Sosa-Rodríguez ${ }^{3}$ \\ Rosa María Galicia-Aguilar ${ }^{4}$ \\ Natalia Ramírez-Girón ${ }^{5}$
}
${ }^{1}$ Doctor en Ciencias de Enfermería. Benemérita Universidad Autónoma de Puebla, Puebla. México. dr.erick.landeros@gmail.com
2 Maestro en Enfermería. Hospital de la Sociedad Española de Beneficencia de Puebla, Puebla. México.
${ }^{3}$ Maestra en Enfermería. Hospital Ángeles de Puebla, Puebla. México.
${ }^{4}$ Maestra en Enfermería. Benemérita Universidad Autónoma de Puebla, Puebla. México.
5 Doctora en Ciencias de Enfermería. Universidad Autónoma de Nuevo León, Monterrey. México.

\section{https://doi.org/10.6018/eglobal.403851}

Recibido: 21/11/2019

Aceptado: 17/02/2020

\section{RESUMEN:}

Objetivo: Describir el efecto de una dosis de ejercicio gradual y progresiva de bajo impacto cardiovascular sobre el autoconcepto físico en un grupo de adultos mayores.

Métodos: Ensayo Clínico Aleatorizado y Controlado con intervención simple ciego en dos grupos equivalentes (experimental y control), bajo un modelo de mediciones repetidas. La muestra estuvo compuesta por 20 sujetos por grupo. Se seleccionaron adultos mayores de 60 a 70 años, de ambos sexos, sin riesgo cardiaco; se excluyeron adultos mayores con patologías que pudieran causar limitaciones en la funcionalidad músculo-esquelética o cardiopulmonar. La intervención consistió en la realización de ejercicio de forma gradual y progresiva, 3 veces por semana durante 10 semanas. El indicador para evaluar el autoconcepto físico fue el Instrumento de Auto descripción (SDQ), con un patrón de respuesta tipo Likert.

Resultados: Ingresaron 45 sujetos, grupo control 22 adultos (edad 66.6 66.14 ) y grupo experimental 23 adultos (edad 67.0 \pm 7.19 ); completaron el programa 38 adultos. En ambos grupos predominaron las mujeres $(66.7 \%)$, nivel socioeconómico medio (73.3\%) y escolaridad básica (44.6\%). No existió diferencia significativa entre los grupos antes de la intervención, en contraste, después de la intervención existió diferencia significativa entre grupos, lo que resultó en un incremento del autoconcepto físico en el grupo experimental y un descenso del autoconcepto físico en el grupo control. Conclusión: La intervención de ejercicio controlado, gradual, progresivo y de bajo impacto cardiovascular, de tres sesiones por semana durante 10 semanas probó ser efectiva para aumentar el autoconcepto físico en el grupo experimental de adultos.

Palabras clave: Ensayo clínico, Ejercicio, Autoconcepto, Adulto Mayor, Teoría de Enfermería. 


\begin{abstract}
:
Objective: To describe the effect of a dose of gradual and progressive exercise of low cardiovascular impact on physical self- concept in a group of seniors.

Methods: Randomized, controlled clinical trial with a blinded experiment in two equivalent groups (experimental one and control one), under a repeated model of measures. The sample consisted of 20 individuals per group. Male and female seniors from 60 to 70 years old with no heart risk, were selected. Seniors with pathologies that could cause limitations on the musculoskeletal function cardiopulmonary functionality were excluded. The intervention consisted of performing exercise gradually and progressively, 3 times a week for 10 weeks. The indicator for assessing physical selfconcept was the Self-Description Questionnaire (SDQ), with a Likert response pattern.

Results: 45 individuals were taken; control group 22 adults (age $66.6 \pm 6.14$ ) and experimental group 23 adults (age $67.0 \pm 7.19$ ); 38 adults completed the program. In both groups, women predominated $(66.7 \%)$, medium socioeconomic level $(73.3 \%)$ and basic schooling $(44.6 \%)$. There was no significant difference between the groups before the intervention, in contrast, after the intervention there was a significant difference between them, which resulted in an increase in physical self-concept in the experimental group and a decrease in physical self-concept in the control group.

Conclusion: The intervention of controlled, gradual, progressive and low cardiovascular impact exercise of three sessions per week for 10 weeks proved to be effective in increasing physical selfconcept in the experimental group of adults.
\end{abstract}

Keywords: Clinical trial, Exercise, Self-concept, Senior Adult, Nursing Theory.

\title{
INTRODUCCIÓN
}

El envejecimiento poblacional es un problema demográfico que representa un reto a los gobiernos e instituciones para adoptar medidas conducentes y atender los efectos del fenómeno. Según la Organización Mundial de la Salud (1) el porcentaje de adultos mayores $(A M)$ en el mundo se duplicará de manera exponencial, pasando de un $12 \%$ en el año 2015 a $22 \%$ en el año 2050. En Latinoamérica, el $11 \%$ de la población actual tiene más de 60 años y se espera que para el año 2030 los AM representen el $17 \%$ de la población general(2). México también presenta un proceso de envejecimiento vertiginoso, los AM pasaron del $6.2 \%$ en el año 2010 a $7.2 \%$ en el año 2015 y se espera que para el año 2050 ésta población aumentará al $16.2 \%{ }^{(3)}$.

El proceso de envejecimiento trae consigo consecuencias importantes de salud; por un lado, se ha documentado predominantemente la inactividad física, dado que el $14.4 \%$ de los AM no practica ejercicio de forma regular ${ }^{(4)}$ lo que favorece el desarrollo de enfermedades crónicas no transmisibles (ECNT) (diabetes, cáncer, eventos cerebro-cardio-vasculares). Por otro lado, se reportan altos porcentajes de alteraciones emocionales como síntomas de ansiedad, depresión, baja autoestima y disminución del auto-concepto físico ${ }^{(5)}$. Adicionalmente, el sistema de salud mexicano no cuenta con programas de actividad física y/o ejercicio para evitar estas consecuencias ${ }^{(6)}$.

A pesar de no existir programas específicos de ejercicio para los AM, se han documentado múltiples beneficios en esta población dado que mejoran el funcionamiento cardiaco, la tensión arterial ${ }^{(7,8)}$, la fuerza funcional ${ }^{(9)}$, favorecen la autonomía $^{(6,10,11)}$, y mejoran el autoconcepto físico ${ }^{(12)}$.

Es aquí donde cobra importancia el uso de marcos teóricos para fundamentar intervenciones en salud, tal como lo indica el Modelo de Adaptación de Callista Roy ${ }^{(13)}$, el cual describe a los seres humanos como sistemas adaptativos que generan conductas (ejercicio físico) a partir entradas (estímulos focal, contextual y residual); las acciones y reacciones producto de estos estímulos se clasifican en cuatro modos de respuesta adaptativos: 1) fisiológico, relacionado con procesos neurales, químicos 
y endocrinos para cubrir las necesidades básicas; 2) rol, que representa la función dentro de la sociedad; 3) interdependencia, basada en la interacción con otros y 4) psicológico, que hace referencia al autoconcepto, definido como el conjunto de creencias y sentimientos que la persona tiene sobre sí misma; está compuesto por sub-áreas que dan dirección a la conducta el yo físico (capacidad de sentir físicamente estímulos externos) y el yo personal (auto-ideal, la auto-consistencia y el yo espiritual-ético-moral).

El autoconcepto general posee componentes específicos para situaciones concretas; para este estudio se considerará el autoconcepto físico (ACF), dado que juega un papel importante en las relaciones interpersonales, en la interacción social, funcionamiento psíquico y la salud de los AM. De acuerdo con Marsh y Shavelson (14), el ACF se divide en dos dimensiones; habilidad física y apariencia física, con nueve dominios: coordinación, actividad física, grasa corporal, físico global, apariencia, fuerza, flexibilidad, resistencia y autoestima. El ACF se ha relacionado positivamente con indicadores de bienestar en los AM como funcionalidad física, afecto positivo y ajuste emocional (12). Sin embargo, la calidad de esta evidencia es limitada por el diseño de los estudios, los cuales, no arrojan resultados contundentes desde el punto de vista experimental, dado que no se ha establecido una dosis de ejercicio específica para valorar el efecto estadístico del ejercicio sobre el ACF en todos sus dominios en población de AM; teóricamente, el profesional de enfermería es el personal de salud idóneo y con las herramientas necesarias para establecer un programa de ejercicio gradual y controlado en los AM que puede contribuir a mejorar el ACF; este mejoramiento puede repercutir en la disminución de padecimientos crónico degenerativos, aumento de la calidad de vida en los AM y un impacto económico positivo en las familias, comunidades y sistema de salud.

Por lo cual se planteó describir el efecto de una dosis de ejercicio gradual progresiva de bajo impacto cardiovascular sobre el autoconcepto físico en un grupo de adultos mayores.

\section{MATERIAL Y MÉTODO}

Ensayo Clínico Aleatorizado y Controlado (ECAC), con intervención simple ciego en dos grupos equivalentes (experimental y control), bajo un modelo de mediciones repetidas. Se seleccionaron Adultos Mayores (AM) de 60 a 70 años, de ambos sexos, sin riesgo cardiaco y que aprobaran las valoraciones de funcionalidad (15,16). Se excluyeron AM con patologías que pudieran causar limitaciones en la funcionalidad músculo esquelética o cardiopulmonar. El muestreo fue probabilístico por técnica de bloques, el tamaño de la muestra fue calculado mediante un programa estadístico con una potencia de prueba de .80 , tamaño de efecto .30 y nivel de significancia de .05 , el tamaño de muestra resultó en 16 sujetos por grupo. Para amortiguar el efecto de atrición la $n$ por grupo fue de 20 sujetos.

En esta investigación se realizó una derivación teórica ${ }^{(17)}$ para explicar la intervención y los resultados de la misma a partir del Modelo de Adaptación de Roy (13) y el abordaje teórico sobre ACF de Marsh y Shavelson ${ }^{(14)}$. El estímulo focal se consideró como la dosis de ejercicio aplicado a los AM para generar modificaciones en el ACF y sus dominios, lo cual hace parte del modo de respuesta adaptativo psicológico (Figura 1). 
Hipótesis. Basado en uno de los postulados de Roy (13), que menciona: "Cuando un modo de respuesta cambia ante un estímulo focal (como el ejercicio) los otros modos o respuestas también se modifican", se formuló la siguiente hipótesis: Si los AM son considerados como un sistema adaptativo al entorno que genera respuestas físicas y psicológicas ante los estímulos, entonces al someter a un grupo de AM a un estímulo focal de actividad física controlada, gradual, progresiva y de bajo impacto cardiovascular, de tres sesiones por semana durante 10 semanas mostrarán una respuesta de adaptación positiva sobre el autoconcepto físico.

Figura 1: Derivación Conceptual Teórico-Empírica

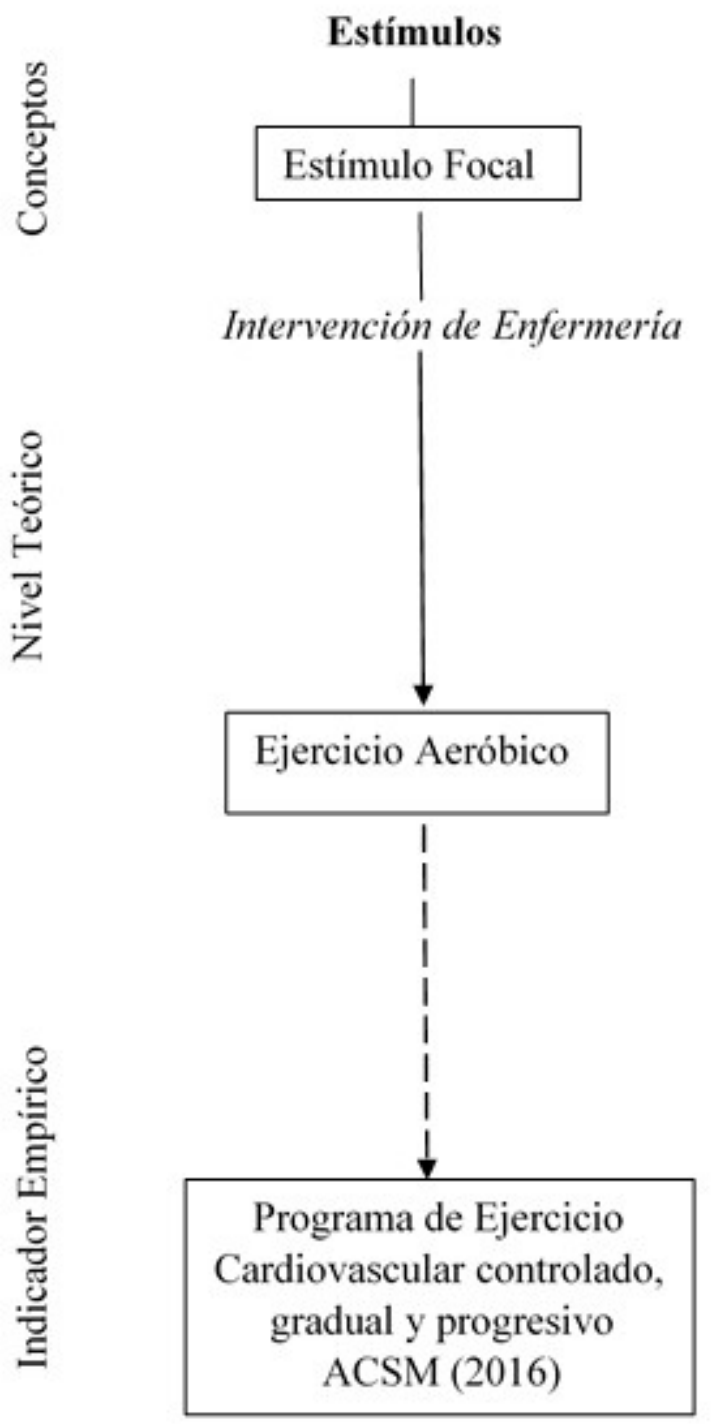

Modos o Respuestas Adaptativas

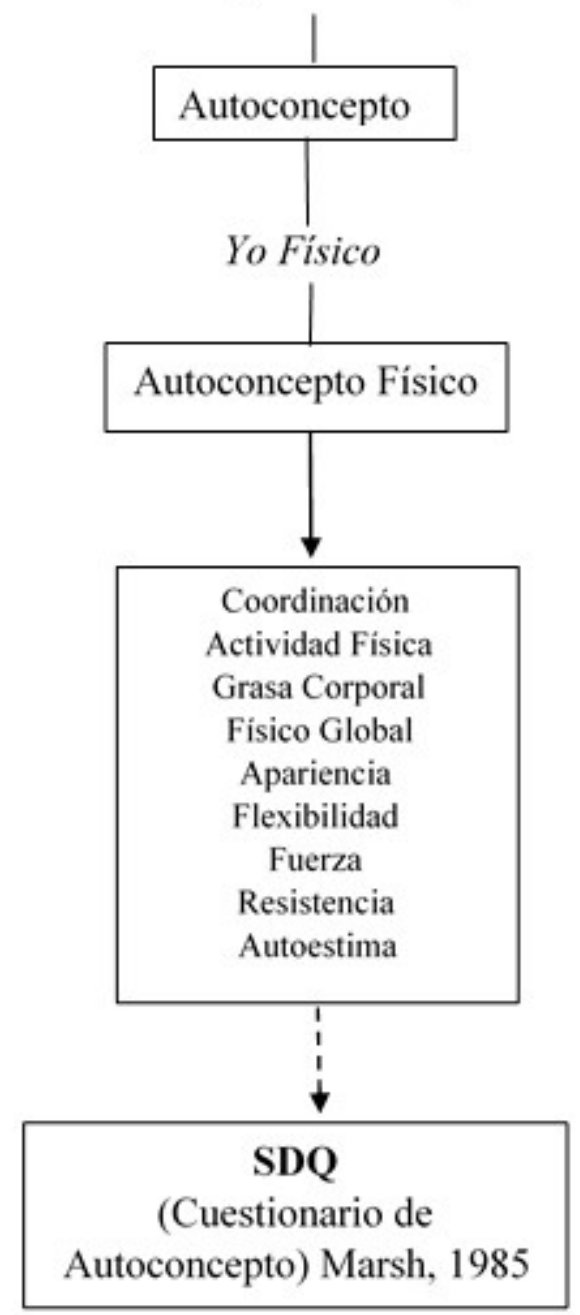

El indicador utilizado para evaluar el ACF fue el Instrumento de Auto descripción SDQ (14), el cual evalúa la apariencia y la habilidad física en 55 reactivos, distribuidos en los dominios: coordinación (reactivos 1, 10, 19, 28, 37,46); actividad física (reactivos 2, 11, 20, 29, 38, 47); grasa corporal (reactivos 3, $12,21,30,39,48$ ); físico global (reactivos 4, 13, 22, 31, 40, 49); apariencia (reactivos 5, 14, 23, 32, 41, 50); fuerza (reactivos 6, 15, 24, 33, 42, 51); flexibilidad (reactivos 7, 16, 25, 34, 43, 52); resistencia (reactivos $8,17,26,35,44,53$ ) y autoestima (reactivos $9,18,27,36,45$, $54,55)$. El patrón de respuesta corresponde a una escala tipo Likert que abarca desde 1= no, 2= más bien no, 3= a veces sí a veces no, 4= más bien sí y 5= sí; siendo el valor máximo 275 puntos y el mínimo 55 puntos, a mayor puntaje mayor será el 
autoconcepto físico percibido. El instrumento ha sido validado en diferentes poblaciones, obteniendo coeficientes alfa de Cronbach entre .81 a .94 (18). El alfa de Cronbach para la presente investigación fue .88.

En relación al procedimiento, para el reclutamiento se repartieron folletos, trípticos y se colocaron lonas en las zonas de mayor afluencia de los AM, donde se indicaron las fechas y horarios de atención para realizar las pruebas de funcionalidad y las valoraciones cardiovasculares que incluyeron los registros del porcentaje de grasa corporal y electrocardiograma, bajo la supervisión de médico certificado. Asimismo, se realizó la aplicación del instrumento SDQ. Las pruebas de funcionalidad y exploración cardiaca fueron realizadas en un espacio tranquilo y cómodo en el área de laboratorio clínico de una Facultad de Salud.

El programa de ejercicio consistió en tres partes: en la primera, se registraron los signos vitales, se hidrataron de acuerdo al peso en kilogramos e iniciaron los ejercicios de calentamiento en dirección cefalocaudal. La segunda parte, los AM realizaron ejercicio de forma gradual y progresiva, iniciaron con 10 minutos y concluyeron con 30 minutos de ejercicio sobre una caminadora, con una frecuencia de tres veces por semana durante diez semanas. El esfuerzo fue calculado gradualmente de $20 \%$ a $50 \%$ de la frecuencia cardiaca de reserva (FCR) (19) y controlado con la velocidad e inclinación de la caminadora. Adicionalmente, los AM identificaron la percepción del esfuerzo físico con la Escala de Borg ${ }^{(20)}$, como un referente clínico al control de signos vitales y auscultación cardiaca mientras estaban sobre la caminadora. En la tercera parte, se realizaron ejercicios de estiramiento y respiración para asegurar que los signos vitales se re-establecieran a parámetros normales, nuevamente fueron hidratados para concluir con la sesión. Los detalles de la dosificación de ejercicio se muestran en la Tabla 1.

Tabla 1. Dosificación de ejercicio aeróbico en AM

\begin{tabular}{|c|c|c|c|c|c|c|}
\hline Semana & Escala de Borg & LPM & ME & FCR & MPH & ICam \\
\hline Semana 1 & 6 muy, muy ligero & $80-90$ & 10 minutos & $20 \%$ & $0.4 \mathrm{mph}$ & $0 \%$ \\
\hline Semana 2 & 6 muy, muy ligero & $90-98$ & 15 minutos & $25 \%$ & $0.6 \mathrm{mph}$ & $0 \%$ \\
\hline Semana 3 & $6-7$ muy ligero & $98-110$ & 20 minutos & $30 \%$ & $0.8 \mathrm{mph}$ & $2 \%$ \\
\hline Semana 4 & 7 muy ligero & $110-119$ & 25 minutos & $35 \%$ & $1.0 \mathrm{mph}$ & $2 \%$ \\
\hline Semana 5 & $7-8$ muy ligero & $119-129$ & 30 minutos & $40 \%$ & $1.2 \mathrm{mph}$ & $2 \%$ \\
\hline Semana 6 & 8 muy ligero & $129-136$ & 30 minutos & $45 \%$ & $1.6 \mathrm{mph}$ & $3 \%$ \\
\hline Semana 7 & $8-9$ muy ligero & $136-142$ & 35 minutos & $50 \%$ & $1.8 \mathrm{mph}$ & $3 \%$ \\
\hline Semana 8 & 9 muy ligero & $136-142$ & 35 minutos & $50 \%$ & $2.0 \mathrm{mph}$ & $4 \%$ \\
\hline Semana 9 & $10-11$ ligero & $136-142$ & 40 minutos & $50 \%$ & $2.2 \mathrm{mph}$ & $4 \%$ \\
\hline Semana 10 & $11-12$ regular & $136-142$ & 40 minutos & $50 \%$ & $2.4 \mathrm{mph}$ & $5 \%$ \\
\hline
\end{tabular}

Nota. LPM. Latidos por minuto, ME. Minutos de ejercicio, MPH. Millas por hora, ICam. Inclinación de la Caminadora. Fuente: Elaboración propia

Para el análisis estadístico, se determinó la curva de distribución normal de la variable dependiente con la prueba de normalidad de Kolmogorov-Smirnov $(p>.05)$, por lo tanto, se utilizó un análisis de varianza de mediciones repetidas (ANOVA MR) y las comparaciones entre grupos con $t$ Student (Figura 2). Se realizaron tres mediciones del ACF, en la semana 0 , semana 5 y semana 10 de la intervención. 
Figura 2: Representación esquemática del plan de análisis estadístico.

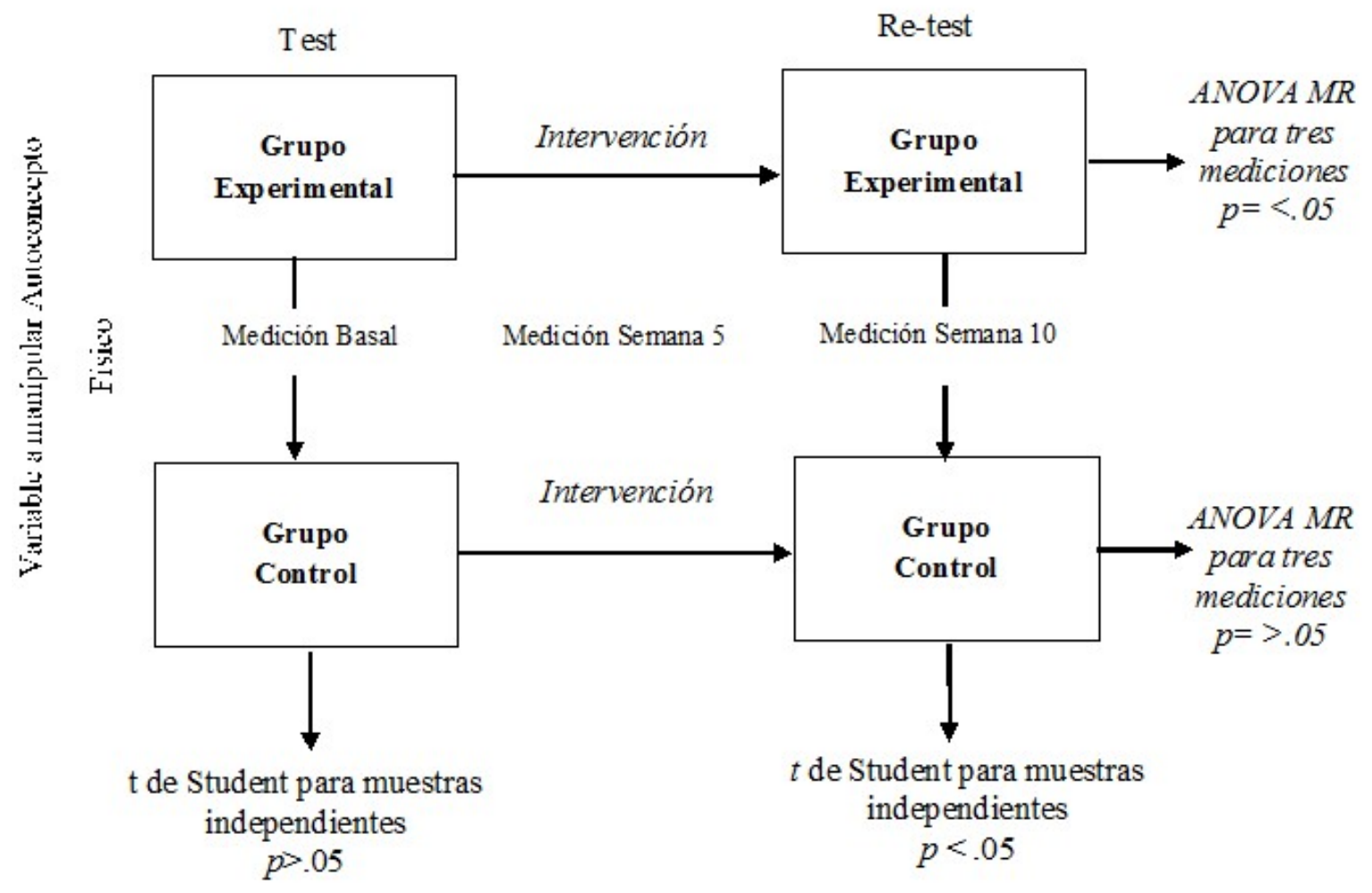

Nota. Esquema de mediciones

Fuente. Elaboración propia

Esta investigación se llevó a cabo de acuerdo a las disposiciones de la Ley General de Salud en materia de investigación (21). Se contó con el consentimiento informado por escrito, se comunicó el objetivo del estudio, la descripción de los procedimientos, beneficios y riesgos; guardando la confidencialidad, privacidad y anonimato. Este trabajo fue avalado por el Comité de Investigación y Estudios de Posgrado de una institución educativa del Estado de Puebla, con el número de registro SIEP/ME/056/2016.

\section{RESULTADOS}

Ingresaron 45 sujetos, para el grupo control $22 \mathrm{AM}$ (edad $66.6 \pm 6.14$ ) y para el grupo experimental $23 \mathrm{AM}$ (edad $67.0 \pm 7.19$ ); completaron el programa $38 \mathrm{AM}$. La progresión del diseño se muestra en la Figura 3. 
Figura 3: Progresión del diseño

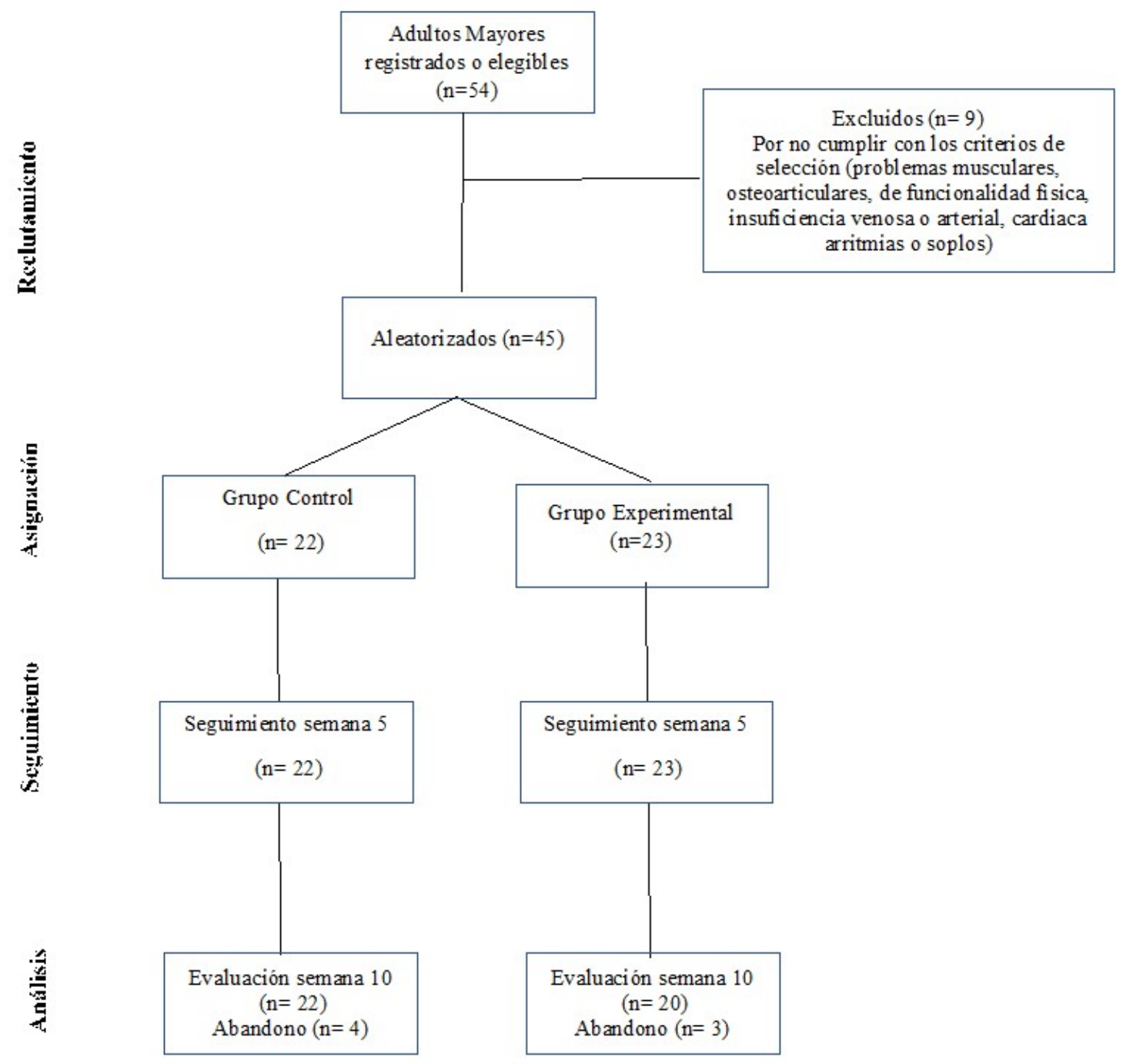

Fuente. Elaboración propia

Las características sociodemográficas fueron equivalentes para los dos grupos; en ambos predominaron las mujeres $(66.7 \%)$, el nivel socioeconómico medio $(73.3 \%)$ y escolaridad básica (primaria y secundaria) (44.6\%); ninguno de los participantes realizaba ejercicio regular al ser seleccionado, la hipertensión arterial (31\%) y la diabetes mellitus $2(26 \%)$ fueron las enfermedades crónicas predominantes. El 37.7\% de los AM mencionaron ser solteros.

Para realizar la contrastación de ambos grupos en el test y en el re-test, se corrieron dos estadísticos $t$ de Student para muestras no relacionadas, cumpliendo a priori los supuestos de normalidad ( $K S=.12, p=.200)$ y homocedasticidad de la varianza a través de la prueba de Levene $(p>.05)$. Los hallazgos indicaron que en el test, no existe diferencia estadísticamente significativa entre los grupos antes de la intervención en el ACF general. En contraste, después de la intervención de ejercicio por diez semanas, existe una diferencia en los promedios y desviaciones estándar en el re-test de ambos grupos de AM.

Se observa un incremento del ACF general en el GE y un descenso de la ACF en el GC. Los datos se presentan en la Tabla 2. 
Tabla 2. Prueba $t$ de Student para contrastación de promedios de grupos no relacionados

\begin{tabular}{c|c|c|}
\hline & \multicolumn{2}{|c|}{ Autoconcepto Físico } \\
\hline & GC (n=22) & GE $(\mathbf{n = 2 3})$ \\
\hline Test & $45.8 \pm 8.0$ & $51.5 \pm 11.1$ \\
& $t=1.95, I C=-.177,11.5, p=.057$ \\
\hline Re-test & $44.0 \pm 6.7$ & $70.4 \pm 16.4$ \\
& $t=6.96, I C=18.7,33.9, p=.001$ \\
\hline
\end{tabular}

Nota: Los promedios aritméticos son presentados con \pm desviación estándar GC= Grupo Control, GE= Grupo Experimental. IC= Intervalos de Confianza al 95\%, gl=grados de libertad (43). Fuente: Elaboración propia

Al realizar la contrastación estadística de las dimensiones del ACF en el test, no se observaron diferencias significativas; sin embargo, al examinar los promedios obtenidos al finalizar la intervención del GE, se observó un cambio en el dominio de apariencia física con el aumento significativo en la dimensión físico global, la apariencia y la autoestima. En el dominio de habilidad física, todas las dimensiones (coordinación, actividad física, fuerza, flexibilidad y resistencia) presentaron un aumento significativo en los promedios aritméticos (Tabla 3).

Tabla 3. Prueba $t$ de Student de re-test del GC y GE, según dominio y dimensión de ACF

\begin{tabular}{|c|c|c|c|c|c|c|}
\hline \multicolumn{7}{|c|}{ Autoconcepto Físico } \\
\hline \multirow[t]{2}{*}{ Dominio } & \multirow[t]{2}{*}{ Dimensión } & \multicolumn{2}{|c|}{ Test } & \multicolumn{2}{|c|}{ Re-test } & \multirow{2}{*}{$\begin{array}{l}\text { t de Student } \\
\text { de re-test }\end{array}$} \\
\hline & & $\begin{array}{c}\text { GC } \\
(n=22)\end{array}$ & GE $(n=23)$ & $\begin{array}{c}\mathbf{G C} \\
(n=22)\end{array}$ & GE $(n=23)$ & \\
\hline \multirow{4}{*}{ 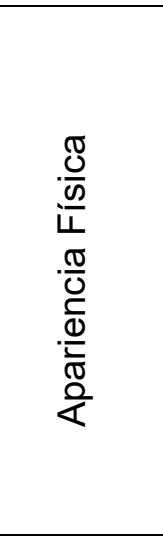 } & $\begin{array}{l}\text { Grasa } \\
\text { corporal }\end{array}$ & $50.5 \pm 23.8$ & $61.4 \pm 30.1$ & $55.6 \pm 24.9$ & $68.8 \pm 26.7$ & $\begin{array}{l}t=1.70, I C=- \\
2.42,28.7 \\
p=.096\end{array}$ \\
\hline & Físico global & $50.9 \pm 17.2$ & $73.9 \pm 20.4$ & $52.4 \pm 15.5$ & $85.3 \pm 20.2$ & $\begin{array}{l}t=6.07 \\
I C=21.9,43.7 \\
p=.001\end{array}$ \\
\hline & Apariencia & $50.0 \pm 14.8$ & $58.1 \pm 20.6$ & $49.0 \pm 15.7$ & $62.5 \pm 23.0$ & $\begin{array}{l}t=2.26, \\
I C=1.49,25.3, \\
p=.028\end{array}$ \\
\hline & Autoestima & $62.0 \pm 19.3$ & $79.5 \pm 18.6$ & $56.0 \pm 18.5$ & $88.1 \pm 12.4$ & $\begin{array}{l}t=6.85, \\
I C=22.7,41.6 \\
p=.001\end{array}$ \\
\hline \multirow{5}{*}{$\begin{array}{l}\frac{\pi}{0} \\
\frac{0}{0} \\
\frac{10}{0} \\
\frac{\pi}{0} \\
\frac{0}{0} \\
\frac{0}{0} \\
\frac{\pi}{1}\end{array}$} & Coordinación & $52.8 \pm 20.1$ & $66.3 \pm 22.2$ & $51.7 \pm 16.8$ & $79.5 \pm 15.3$ & $\begin{array}{l}t=5.79, I C=- \\
18.1,37.5 \\
p=.001\end{array}$ \\
\hline & $\begin{array}{l}\text { Actividad } \\
\text { Física }\end{array}$ & $25.7 \pm 18.6$ & $25.7 \pm 23.4$ & $22.9 \pm 18.2$ & $55.4 \pm 28.5$ & $\begin{array}{l}t=4.53, \\
I C=18.5,46.9 \\
n=001\end{array}$ \\
\hline & Fuerza & $49.6 \pm 14.8$ & $61.6 \pm 21.3$ & $46.5 \pm 11.9$ & $67.9 \pm 24.2$ & $\begin{array}{l}t=3.72, \\
I C=9.77,32.9, \\
p=001\end{array}$ \\
\hline & Flexibilidad & $40.5 \pm 17.4$ & $58.9 \pm 22.8$ & $38.3 \pm 12.5$ & $69.9 \pm 34.7$ & $\begin{array}{l}t=4.00 \\
I C=15.6,47.3, \\
p=.001\end{array}$ \\
\hline & Resistencia & $27.6 \pm 13.8$ & $39.3 \pm 23.9$ & $21.6 \pm 14.4$ & $53.0 \pm 29.4$ & $\begin{array}{l}t=4.46, \\
I C=17.0,45.1 \\
p=.001\end{array}$ \\
\hline
\end{tabular}


En la tabla 4, se muestran los valores de las comparaciones múltiples entre las tres mediciones del GE (Prueba Post Hoc/Tukey). Se observaron cambios significativos de la medición uno en comparación con las mediciones dos y tres $(p<.05)$. Sin embargo, los valores de la segunda medición en comparación con la tercera, no presentan diferencia significativa. En el GC no se observa significancia estadística (los datos no se muestran).

Tabla 4. Prueba Post hoc- Tukey para comparación de ACF del GE

\begin{tabular}{ccccccc}
\hline $\begin{array}{c}(\mathbf{I}) \\
\text { Medición }\end{array}$ & $\begin{array}{c}(\mathbf{J}) \\
\text { Mediciones }\end{array}$ & $\begin{array}{c}\text { Diferencia } \\
\text { de medias } \\
(\mathbf{I}-\mathbf{J})\end{array}$ & $\begin{array}{c}\text { Error } \\
\text { típico }\end{array}$ & Sig. & \multicolumn{2}{c}{ IC 95\% } \\
& 2 & -11.64 & 2.93 & .002 & -19.2 & -4.05 \\
& 3 & -18.90 & 3.73 & .001 & -28.5 & -9.23 \\
& 1 & 18.90 & 3.73 & .001 & -9.23 & 28.5 \\
3 & 2 & 7.25 & 3.63 & .175 & -2.15 & 16.6 \\
\hline
\end{tabular}

Nota: Medición 1 (semana 0), Medición 2 (semana cinco), Medición 3 (semana diez).

Fuente: Elaboración propia

\section{DISCUSIÓN}

El objetivo del estudio consistió en describir el efecto de una dosis de ejercicio gradual progresiva de bajo impacto cardiovascular sobre el autoconcepto físico en un grupo de adultos mayores. La intervención de ejercicio tuvo como base el Modelo de Adaptación de Roy (13) y se apoyó en los conceptos de autoconcepto físico de Marsh ${ }^{(14)}$. Se examinaron dos grupos de AM cuyas características personales fueron equivalentes en edad, estado civil y nivel socio económico, la mayoría de los participantes en ambos grupos fueron mujeres entre 60 y 70 años de edad, gran parte de la muestra presentó hipertensión arterial. El efecto principal de la dosis de ejercicio fue un aumento general en el ACF durante el programa de ejercicios de 10 semanas en el GE.

Con relación a los postulados teóricos de Roy ${ }^{(13)}$, el ejercicio cardiovascular controlado como estímulo focal y como hábito permanente, mostró mejorar y aumentar el ACF, tal como lo reportó Bohórquez et al. (6), quienes no encontraron diferencias significativas entre hombres y mujeres. Adicionalmente, otros autores han reportado la importancia de esta variable cognitiva como predictora y determinante para el mantenimiento de la salud física y mental en los AM como medio para lograr una mejor calidad de vida ${ }^{(22,23)}$.

En relación al modo de respuesta psicológico determinado por el ACF en $A M$, se determinó el incremento en los dominios de apariencia física y habilidad física. En la primera, las dimensiones que tuvieron incremento estadísticamente significativo fueron el físico global, la apariencia y la autoestima; la mejoría en estas dimensiones es consistente con la literatura publicada ${ }^{(22)}$, dado que la realización de actividad física en los AM tiene efectos antiinflamatorios, antioxidantes, mejora la vascularización facilitando un mejor color y calidad de la piel y del cabello, menor retención de líquidos, mejora el sistema inmunológico, disminuye la secreción de insulina, mejora el ritmo intestinal y en general los AM tienen una mayor salud funcional, lo que favorece en gran medida la apariencia y repercute positivamente en el aumento de la autoestima. 
Respecto al segundo dominio, todas las dimensiones (coordinación, actividad física, fuerza, flexibilidad, resistencia) presentaron mejoría significativa en consistencia con la literatura, dado que el ejercicio físico mejora el funcionamiento del sistema nervioso, lo que representa menor riesgo de caídas, funciones cognitivas mejor conservadas y menor riesgo de limitaciones funcionales moderadas y graves; lo cual permite al AM mejorar la realización de las actividades básicas e instrumentales de la vida diaria ${ }^{(8-10)}$.

El cambio en ambos dominios del ACF se presentó a partir de la semana número cinco, tal como lo reportó Cardona et al. (10) después de una intervención de 150 minutos, tres veces a la semana durante 10 semanas, basada en ejercicio respiratorios y aeróbicos. Específicamente, López ${ }^{24)}$ reportó un aumento en la fuerza muscular después de realizado un programa de ejercicios de 30 minutos durante 8 semanas. En contraste, otros autores como Calero et al. (22) tardaron seis meses en obtener resultados significativos. Los hallazgos del ensayo clínico muestran que para probar el efecto del ejercicio sobre el ACF de los AM, no es necesario 10 semanas de ejercicio, solo se requiere la mitad.

El estudio presentó algunas condiciones que pudieron limitar los resultados obtenidos, tales como: no contar con mayor número de facilitadores para generar sesiones vespertinas y probar si hay diferencia entre horarios (adherencia) para realizar ejercicio, dado que la literatura refleja que el horario matutino es mucho mejor para la adherencia del AM en relación a la dosis de ejercicio; no se realizó la medición adicional de variables tales como glucosa, tensión arterial y el peso para poder probar si con esta dosis de ejercicio pueden mejorar también otras variables físicas y poder analizar otras variables cognitivas; no se realizaron ejercicios de fuerza y repeticiones para evitar que los AM presentaran problemas cardiacos.

\section{CONCLUSIÓN}

La intervención de actividad física controlada, gradual, progresiva y de bajo impacto cardiovascular, de tres sesiones por semana durante 10 semanas, probó ser efectiva para aumentar el ACF y sus dominios en el grupo experimental de adultos mayores. Dicho resultado prueba la hipótesis de este estudio a través del postulado del modelo de adaptación de Roy ${ }^{(13)}$, el cual establece que los AM son un sistema adaptativo al entorno que genera respuestas físicas y psicológicas ante diferentes estímulos.

\section{REFERENCIAS}

1. Organización Mundial de la Salud. Envejecimiento y salud, Notas descriptivas 2018. Disponible en: http://www.who.int/es/news-room/factsheets/detail/envejecimiento-y-salud

2. Federación Iberoamericana de Asociaciones de Personas Adultas Mayores FIAPAM. America Latina envejece a pasos de gigantes. 2019. Disponible en: https://fiapam.org/america-latina-envejece-a-pasos-de-gigante/

3. Instituto Nacional de Estadística, Geografía e Informática. Proyecciones de la población de México 2010-2050. Recuperado de: http://www3.inegi.org.mx/sistemas/sisept/Default.aspx?t=mdemo249\&s=est\&c=339 
4. Instituto Nacional de Salud Pública. Encuesta Nacional de Salud y Nutrición de Medio Camin. Informe Final de Resultados. Secretaria de Salud de México, 2016.

5. Santana YDLCB., Enríquez JB, Rodríguez YM, Díaz AS. Determinación de los factores psicosociales potenciadores de conductas suicidas en los adultos mayores. MediCiego [Internet]. 2015 [citado 15 septiembre 2019]; 21(1). Disponible en: http://www.revmediciego.sld.cu/index.php/mediciego/article/view/85

6. Bohórquez MR, Lorenzo M, García AJ. Actividad física como promotor del autoconcepto y la independencia personal en personas mayores. Revista Iberoamericana de Psicología del Ejercicio y el Deporte [Internet]. 2014 [citado 15 septiembre 2019]; 9(2), 533-546. Disponible en: https://idus.us.es/xmlui/handle/11441/59364

7. Hernández $\mathrm{O}$, Ramírez F. Programa de ejercicio estructurado es viable y mejora la capacidad funcional en adultos mayores en Puerto Rico. Rev Ciencias del Ejercicio y Salud [Internet]. 2014 [citado 20 septiembre 2019]; 12(2). 1-15. Disponible en: http://repositorio.ucr.ac.cr/handle/10669/21645

8. Wehmeyer C, Loots JM, Nortjé, L, Lategan, L. The effects of supervised exercise training and self -directed active daily living on fitness and health parameters in elderly males with type II diabetes. African journal for physical, Health education, recreation and dance [Internet]. 2014 [citado 20 septiembre 2019]; 20(4:1). 1424-1435 9. Rodríguez-Berzal E, Aguado-Jódar X. Efectos del entrenamiento de la fuerza funcional en persons mayores. Apunts [Internet]. 2015 [citado 20 septiembre 2019]; 10(09). 64-71. Disponible en: https://www.sciencedirect.com/science/article/abs/pii/S1886658115000304

10. Garcia, NC, Ramirez JCG, Ypia MAT, Tonguino-Rosero S. Efecto de un programa de ejercicios respiratorios y aeróbicos en medio acuático versus terrestre para adultos mayores. Revista salud UIS [Internet]. 2016 [citado 20 septiembre 2019]; 48(4).

Disponible

en:

https://revistas.uis.edu.co/index.php/revistasaluduis/article/view/5835

11. García FG, Angulo J. Análisis de rasgos de personalidad positiva y bienestar psicológico en personas mayores practicantes de ejercicio físico vs no practicantes. Revista Iberoamericana de Psicología del Ejercicio y el Deporte [Internet]. 2016 [citado 20 septiembre 2019]; 11(1). 113-122. Disponible en: https://scholar.google.com.mx/scholar?hl=es\&as sdt=0\%2C5\&q=An\%C3\%A1lisis+de +rasgos+de+personalidad+positiva+y+bienestar+psicol\%C3\%B3gico+en+personas+ mayores+practicantes+de+ejercicio+f\%C3\%ADsico+vs+no+practicantes\&btnG=

12. Infante G, Goñi A, Villaroel JD. Actividad física y Autoconcepto Físico y general a lo largo de la edad adulta. Revista de Psicología del Deporte [Internet]. 2011 [citado 20 septiembre 2019]; 20(2), 429-444

13. Roy C. The Roy Adaption Model. Elements of the Roy Adaptation Model. University of Alberta. City of Toronto. Third Ed. Pearson. Chapter 2, 2009.

14. Marsh H, Shavelson R. Self-concept: Its multifaceted hierarchical structure. Educational Psychologist [Internet]. 1985 [citado 20 septiembre 2019]; 20(3), 107-123.

15. Katz S, Stroud MW. Functional assessment in geriatrics. A review of progress and directions. Journal of American Geriatric Society [Internet]. 1989 [citado 20 septiembre 2019]; 3(37), 267-271. Disponible https://onlinelibrary.wiley.com/doi/abs/10.1111/j.1532-5415.1989.tb06820.x

16. Tinetti ME, Baker D, King M. Effect of Dissemination of Evidence in Reducing Injuries from Falls. The New England Journal Medicine [Internet]. 2008 [citado 20 septiembre 2019]; 359(3), 252-61. Disponible https://www.nejm.org/doi/full/10.1056/NEJMoa0801748 
17. Walker LO, Avant KC. Strategies for Theory Construction in Nursing (Quinta ed.). Texas: Prentice Hall, 2011.

18. Leach. LF, Henson RK, Odom L, Cagle LS. A reliability generalization study of the Self-Description Questionnaire. Educational and Psychological Measurement [Internet]. 2006 [citado 20 septiembre 2019]; 66, 285-304.

19. American College of Sports Medicine. ACSM,s. Guidelines for Exercise Testing and Prescription. $8^{\circ}$ Edition Lippincott Williams \& Wilkins. USA, 2010.

20. Borg G. Borg Rating of Percived Exertion (RPE) Scale. (c) Gunnar Borg, 1970, 1985, 1994, 1998.

21. Cámara de Diputados del Honorable Congreso de la Unión.Ley General de Salud. Última reforma publicada DOF 04-06-2014. Mexico D.F, Mexico. Disponible en: http://www.diputados.gob.mx/LeyesBiblio/pdf/142_040614.pdf

22. Calero S, Klever T, Ramiro M, Rodríguez A, Analuiza E. Influencia de las actividades físico-recreativas en la autoestima del adulto mayor. Revista Cubana de Investigaciones Biomédicas [Internet] 2016 [citado 20 septiembre 2019]; 35(4). 266$374 . \quad$ Disponible en: http://scielo.sld.cu/scielo.php?pid=S0864$03002016000400007 \&$ script $=$ sci arttext\&tlng=pt

23. Marcos P, Orquín F, Belando N, Moreno J. Motivación autodeterminada en adultos mayores practicantes de ejercicio físico. Cuadernos de Psicología del Deporte [Internet] 2014 [citado 20 septiembre 2019]; 14(3).149-156. Disponible en: https://revistas.um.es/cpd/article/view/211411

24. López JC, Arango EF. Efectos del entrenamiento en superficies inestables sobre el equilibrio y funcionalidad en adultos mayores. Rev Fac Nac Salud Pública [Internet] 2015 [citado 20 septiembre 2019]; 33(1):31-39.Disponible en: http://tesis.udea.edu.co/handle/10495/4493 